\title{
The Study on the Development of Decision Support Systems in Response to Catastrophic Social Risks
}

\author{
Xie Zhenhua \\ School of Public Administration \\ Xiangtan University \\ Xiangtan, Hunan, China \\ E-mail: wuqingxiezhenhua@126.com
}

\author{
He Zhen \\ School of Public Administration \\ Xiangtan University \\ Xiangtan, Hunan, China \\ E-mail: hezhen6984@aliyun.com
}

\begin{abstract}
After the catastrophe, the key to whether the government can effectively deal with and control social risks caused by the disaster lies in whether to make the right decisions under the unified leadership and the unity of command. Therefore, the establishment of Decision Support System (DSS) in response to catastrophic social risks is the point to risk management. Through the development of DSS to deal with catastrophic social risk, it can take full advantage of decisive information to deal with catastrophic social risk by strengthening the construction of the data warehouse and building a variety of powerful model library and method library. That DSS makes government organizations, non-profit organizations, citizens, enterprises and other agents form a crisis decision-making force as a whole. Then, dynamic cooperation networks will substitute mechanical and rigid organizational level in traditional governmental organizations. Then scientific decision-making will be realized.
\end{abstract}

\section{Key Words: Catastrophe; Social risk; DSS}

\section{INTRODUCTION}

The mechanism in response to catastrophic social risks includes two processes, namely the decision-making process and the implementation process. In current mechanism, China has a certain advantage in the rapid concentration of resources, the allocation of the deployment and organizational implementation. But there are problems in the decision-making process: First, the system to collect and analyze the early signs that appears in the decision-making process in response to catastrophic social risks has large defects. Second, the current response mechanism has disadvantage in aggregating and determinating the information when the catastrophe bursts. Third, the current response mechanisms of decision-making process evolution trends and impact analysis and determined system are defective. According to the theory of complex adaptive systems, developing advanced DSS in response to catastrophic social risks and dynamically simulating catastrophic social risks become an indispensable important technique in supporting government decision-making.

\section{CONSTRUCTION OF CATASTROPHIC SOCIAL RISKS \\ DATA WAREHOUSE}

Data warehouse strips, extracts and converses a large number of traditional database datum that are used to clean up things, and then the data is re-organized in the need of decision-making theme[1]. Logical structure of the data warehouse can be divided into recent basic data layer, historical data layer and integrated data layer. The physical structure of the data warehouse generally uses star-schema relational database. Star-schema database represents spatial multi-dimensional cube. This highly concentrated database provides useful analysis for various decision-making demands. With the development of the data warehouse, Data Mining technology has also developed rapidly. Data warehouse focuses on the storage and management of data that orients to decision-making agent, while Data Mining pays more attention to the analysis of data in data warehouse, and then transforms it into decision-making assistant information.

DDS to deal with catastrophic social risks requires a lot of information support. Especially it should pay more attention to the signs that appears in early catastrophic social risk. In order to search and analyze the information, it is necessary to follow information and collect a variety of basic information, such as remote sensing information, social information, geographic information, economic information and other basic information. When catastrophe happens, DDS requires to collect and aggregate catastrophic alarm information, image information, spatial information, supplying resources information and other supporting information. Through construction of data warehouse(Figure 1), it will be possible to realize real-time collection and standardized process, which will provide data support for DDS.

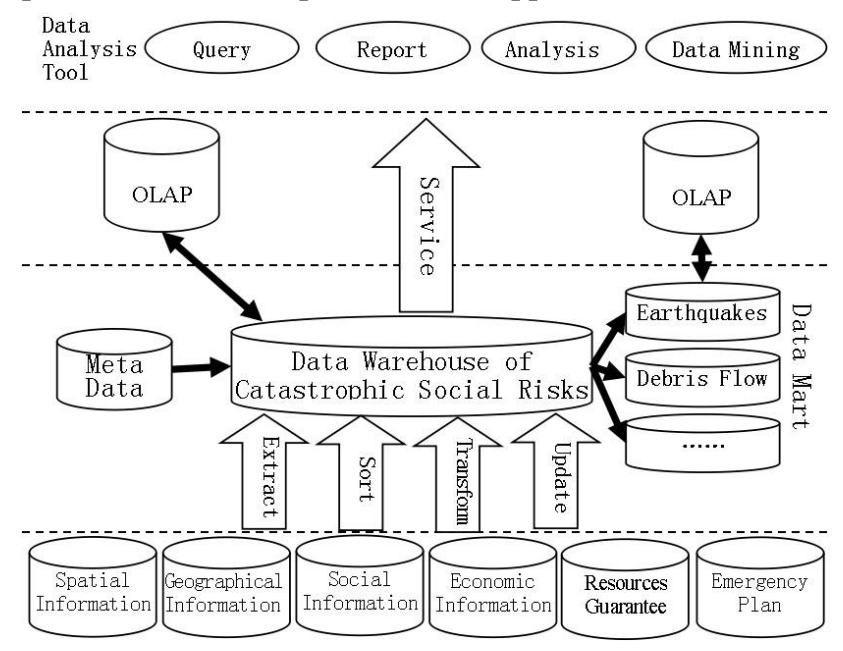

Figure 1. Data Warehouse of Social Catastrophe Risk

\section{CONSTRUCtion OF Decision MOdEl Database IN}

\section{RESPONSE TO CATASTROPHIC SOCIAL RISKS}

Served as one of the three pillars of traditional DDS, model base system is one of the most distinctive parts of the system[2]. DDS can provide effective support during 
the decision-making process, mainly because model base system provides decision-makers with reasoning, comparisons, selections, and analysis to the overall problems. Therefore, model base system and its corresponded model base management have played significant roles in DDS. In the process of response as well as decision-making to catastrophe social risks, the models we need to construct include:

\section{A. Information Retrieval Model in Response to Catastrophic Social Risks}

Since occurrence and response of catastrophe is always in a dynamic environment, it is necessary to establish the scientific information retrieval model so as to immediately grasp the relevant information(Figure 2).In general, when the uncertainty of decision-making increases, the difficulty in obtaining and analyzing information will increase too[3].The approach to obtain information will change accordingly. Decision-makers are required to obtain more relevant information from natural environment, social environment, and even social network. For example, after Wenchuan Earthquake, the communication facilities have been destroyed. A lot of information spread through QQ, MSN and other social networks.

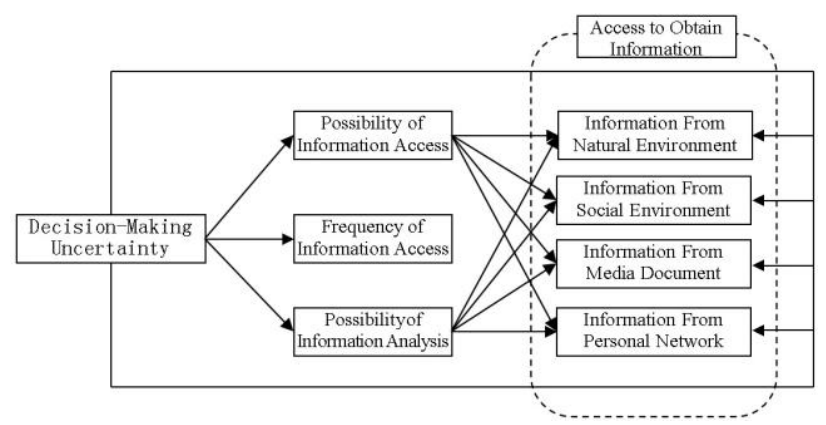

Figure 2. Information Retrieval Model in response to Catastrophic Social Risks

\section{B. Multi-attribute Decision Model Based on \\ Multi-objective Genetic Algorithm}

Social risk decision-making which caused by catastrophe, need to consider several objectives. In order to find the Pareto optimal solution, ways to improve the bad stratified multi-objective genetic algorithm can be adopted[4].The model uses the decision-making matrix that is made of the Pareto optimal solution, to obtain the attributes weights by the method of information entropy, and then uses the ordinal preference method which approximates the ideal solution (TOPSIS) to sequence the Pareto optimal solutions.

\section{Regulation Models of Catastrophic Social Risk}

After the catastrophe, the government implements measures to reduce the risk of catastrophe on the basis of the judgment after acquiring relevant information and reviewing the cause, the event properties, and the potential scale of impact of the catastrophe[5]. However, in the process of decision-making, even if the government timely launches a emergency plan, and takes actions promptly, as for the trends of the events, there still exists a natural lags. The regulation models of risk mainly aim to find out the right time to take appropriate action, and to reduce social risk with minimum cost. According to the influence trend of social risk of catastrophe, we can classify these models into three types: outright type, sudden type and leisure type. This project studies and makes a comprehensive comparison of the three types including the duration of trend evolution and regulation, as well as the loss cost of trend evolution ,the input cost of trend regulation, and the comprehensive cost of trend control, which contributes to the systematic construction of optimal decision.

\section{CONSTRUCTION OF DECISION-MAKING METHOD \\ LIBRARY IN RESPONSE TO CATASTROPHIC SOCIAL RISKS}

A method is modular programming unit with specific functions. Specifically, it is a process of the algorithm. Method and model in the nuclear emergency decision-making activities are mutually supportive. Methods can also be defined as various analytic and tectonic tools for users to model problems[6]. Mainly they refer to a variety of algorithms, solution and equation involved in models. By implementing these algorithm, a specific model and its parameters can be determined. Method library is a collection of methods that can be expanded. It can also be shared and different model can call the same method. Method library includes two parts. One is a collection of methods; the other one is intelligent information of using method. It can solve the problem to choose method. Exactly which method to choose in decision-making responsing to catastrophic social risks depends on concrete conditions. The method is determined by the information that is transmitted from users, data warehouse and method library. Decision-making method library in response to catastrophic social risks mainly includes: multi-attribute utility analytic method, Analytic Network Process and group decision- making method based on Bayesian theory, etc.

\section{A. Multi-attribute Utility Analytic Methods}

In decision-making responsing to catastrophic social risks, a utility function with scoring can be derived from all the factors that is relative to decision-making scheme. Multi-attribute utility function can be expressed as in (1):

$$
U_{j}=\sum_{i=1}^{n} w_{i} u_{j i}
$$

Where, $\mathrm{j}$ is the program number, $\mathrm{i}$ is the attribute number, $U_{j}$ is the utility value of the $j$-th program, $W_{i}$ is the weight of the $i$-th attribute, $U_{j i}$ is the property value of the $\mathrm{i}$-th attribute in the $\mathrm{j}$-th program. When there are two or more than two options to choose, the solution with maximum points(utility value)is the optimal solution.

\section{B. Analytic Network Process}

Analytic Network Process (ANP) is a decision-making method to adapt to non-independent hierarchical structure, which is proposed by Professor T.L.Saaty in 1996. He works in University of Pittsburgh. This method is a new practical decision-making method that is derived from 
Analytic Hierarchy Process (AHP)[7]. AHP provides a basic method to measure decision-making factors as a decision-making process. ANP firstly divides element of decision-making system into two parts: The first part is called the controlling factor layer, including decision-making objectives and decision criteria. Each weight of criterion in control layers can be obtained by AHP. The second part is the network layer, It is a network structure which is made by all the element groups in the dominance of control layer. Elements mutually depend and dominate. The internal between element and layer is independent. In hierarchical structure, what is dominated by each criterion is not a simple independent element, but a mutually dependent network structure. This method will help to make scientific decision when complex problems occur.( Figure 3).

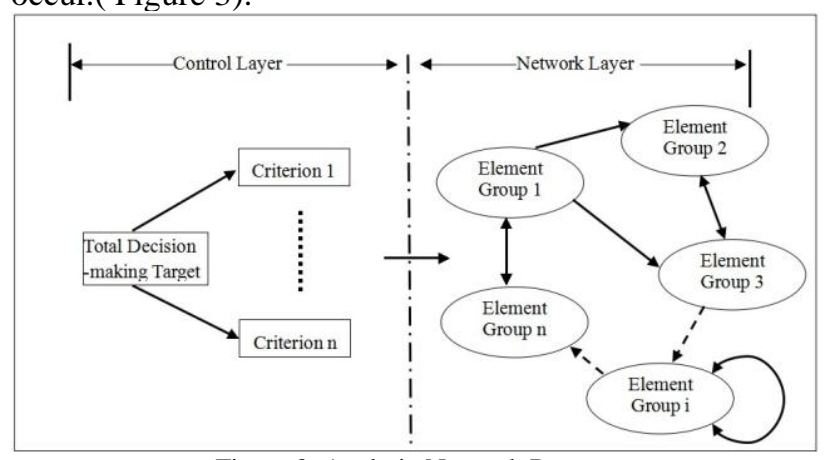

Figure 3. Analytic Network Process

\section{Group Decision-making Method Based on}

\section{Bayesian theory}

When making decision in response to catastrophic social risks, different decision-maker has different choice. Their preference will be considered as random variables. By using regression model and Bayesian analysis method to deduce the posterior distribution of group judgment, which depend on the multiplier to determine matrix, the preference of group will be obtained. Then Gibbs sampling algorithm based on MCMC (Markov chain Monte Carlo method) will compute the random preference information that exists in decision-making matrix. Its posterior distribution will be get. Since large preference deviation exists in large group. It is necessary to divide large group into different small groups according to the posterior preference deviation of decision-making group. Then the sub-group who has same preference will form together in order to further reach agreement between group. If the decision-making scheme $\mathrm{x}$ gets ranked $\mathrm{r}$, the acceptable factor will be $p_{i}^{r}$.Then the overall ranking certainty factor of scheme $\mathrm{i}$ will be expressed as in (2):

$$
a_{i}^{h}=\sum_{r=1}^{n} \omega_{r} p_{i}^{r}
$$

$\left(\omega_{r}\right.$ ranking weight). Different decision-maker who has different risk preference will give different $\omega_{r}$. For the people who like risks, the $\omega_{r}$ will be get through the schema that is expressed as in (3):

$$
\omega_{r}=\frac{1}{r^{c}}-\frac{\sum_{r=1}^{n} 1 / r^{c}}{n}
$$

If $\mathrm{C}$ becomes greater, it means that decision-makers are more willing to take risks. For the people who avoid risks, the $\omega_{r}$ will be get through the schema that is expressed as in (4):

$$
-\frac{1}{(n+1-r)^{c}}+\frac{\sum_{r=1}^{n} 1 /(n+1-r)^{c}}{n}
$$

If $\mathrm{C}$ becomes greater, it means that decision-makers are more willing to avoid risks. For the risk-neutral decision-makers, they can consider the average of the two equations as ranking weights.

\section{COnStruction OF MUlti-AgEnt Based DDS IN}

\section{RESPONSE TO CATASTROPHIC SOCIAL RISK}

Due to the uncertain objective world and limited human rationality, social risk trigged by catastrophe is always accompanied with the development of society. Especially in modern society, the development of science and technology gives more variables and parameters to the social life that has been full of uncertainty, thus leading to its highly unstable and uncertain state all the time[8]. Crisis and risk have been the normality of social environment. So it will set a still higher demand on public crisis decision-making. In process of public crisis decision-making, it is no doubt that a single organization will have difficulty in fully understanding every aspects and various technique of decision-making. So it is necessary to maximize pluralistic governance agent to form decision-making network system. By researching on the position and the role of multiple decision-makers, including core-status government organizations, citizens, enterprises, as well as international organizations, in response to social crisis, this project tries to establish more efficient, more rapid DSS, utilizing modern information networks and cloud computing environments. (Figure 4). In this system, although each of main body in decision-making process is distributed in every corner of society, they will interact frequently and form overall strength to handle crisis, instead of inflexible, rigid administrative hierarchy operated by traditional governments, new main body will take advantage of dynamic collaboration networks to make decisions more scientifically.

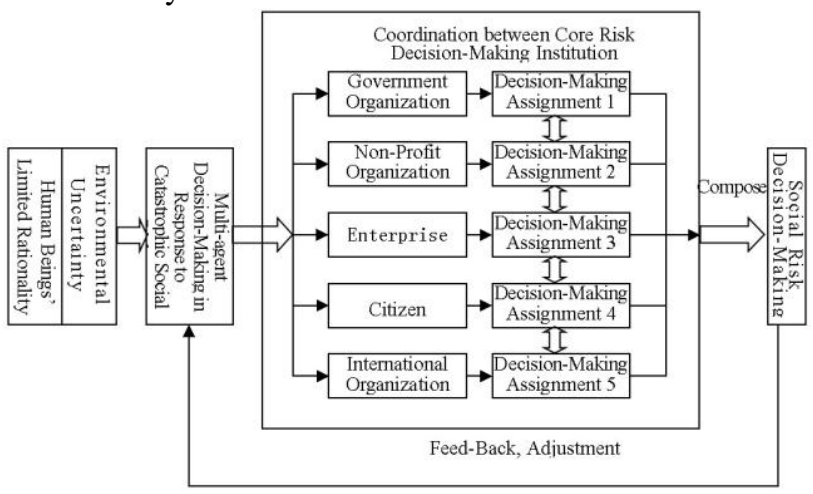

Figure 4. DDS in Response to Catastrophic Social Risks Based on Multi-agent

\section{CONCLUSION}

By exploring the key supporting technologies of DSS and the study of standardization, automation, intelligence, facilitation of DSS in response to catastrophic social 
risks, it effectively improves the ability of emergency management at all levels of government in the face of catastrophe and related social problems and helps to improve the effective disposal capacity of the government in disaster prevention and emergency response, to enhance the government's administrative ability, to promote the construction of service-oriented government.

\section{ACKNOWLEDGMENT}

This article is supported by two programs. One is "The Study on Information Disclosure Mechanism of Local Government in Response to Emergency", which belongs to New Century Excellent Talents in University. Its program number is NCET-10-0167. The other one is "The Study on the Evolution Rule and Response Decision-making of Catastrophic Social Risks", which supported by Hunan provincial innovation foundation for postgraduate. Its program number is CX2013B244.

\section{REFERENCES}

[1] Ji,Min Jin,Fengyang Li,Ting etc. Construction of Marine multi-dimensional data warehouse. Oceanography (Chinese edition), Article in a journal:2009, (6): 48-53.

[2] Fan, Bo Government Intelligence: Decision Support Technology of Government Active Service Model, Beijing: Tsinghua University Press , 2006

[3] Petter S, Fruhling A. Evaluating the success of an emergency response medical information system. International Journal of Medical Informatics, Article in a journal:2011,80(7) .

[2] Tang, X., et al., The Shanghai Multi-Hazard Early Warning System: Addressing the Challenge of Disaster Risk Reduction in an Urban Megalopolis, in Institutional Partnerships in Multi-Hazard Early Warning Systems, M. Golnaraghi, M. Golnaraghi^Editors. 2012, Springer Berlin Heidelberg. pp. 159. Article in a conference proceedings:

[5] Wang Weiguo, etc. Construction of Portal-based Integrated Information Portal for Avian Influenza. Application Research of Computers, Article in a journal:2007(7). vol.24.pp.279-282

[6] Fan Weicheng,A Study on the Support System Construction of the Country's Public Safety and Emergency Management China Emergency Management, Article in a journal:2008.4.

[7] WANG Dao-ping,LIU Li-xian,LI Peng. Research on Cooperative Relationship between Supply Chain Members based on ANP, Soft Science, Article in a journal:2011 (5)31-36.

[8] Chen, Yuanzhang Researches on Social Risk Management of Unexpected Events of China in Transition Central South University PhD thesis, 2009. 